\title{
Nonplanar on-shell diagrams and leading singularities of scattering amplitudes
}

\author{
Baoyi Chen ${ }^{2}$, Gang Chen ${ }^{1,2, a}$, Yeuk-Kwan E. Cheung ${ }^{2}$, Yunxuan $\mathbf{L i}^{2}$, Ruofei Xie ${ }^{2}$, Yuan Xin ${ }^{2}$ \\ ${ }_{1}^{1}$ Department of Physics, Zhejiang Normal University, Jinhua, Zhejiang, People's Republic of China \\ ${ }^{2}$ Department of Physics, Nanjing University, 22 Hankou Road, Nanjing 210093, People's Republic of China
}

Received: 30 September 2016 / Accepted: 31 January 2017 / Published online: 8 February 2017

(C) The Author(s) 2017. This article is published with open access at Springerlink.com

\begin{abstract}
Bipartite on-shell diagrams are the latest tool in constructing scattering amplitudes. In this paper we prove that a Britto-Cachazo-Feng-Witten (BCFW) decomposable on-shell diagram process a rational top form if and only if the algebraic ideal comprised the geometrical constraints are shifted linearly during successive BCFW integrations. With a proper geometric interpretation of the constraints in the Grassmannian manifold, the rational top form integration contours can thus be obtained, and understood, in a straightforward way. All rational top form integrands of arbitrary higher loops leading singularities can therefore be derived recursively, as long as the corresponding on-shell diagram is BCFW decomposable.
\end{abstract}

\section{Introduction}

Scattering amplitudes are of profound importance in high energy physics describing the interactions of fundamental forces and elementary particles. The scattering amplitudes are widely studied for $\mathcal{N}=4$ super Yang-Mills theory and QCD. At tree level, BCFW recursion relations [1-4] can be used to calculate $n$-point amplitudes efficiently. Unitarity cuts [5-7] and generalized unitarity cuts [8-15] combined with BCFW for the rational terms work well at loop level [16-21].

Leading singularities [22] are closely related to the unitarity cuts of loop-level amplitudes. For planar diagrams in $\mathcal{N}=4$ super Yang-Mills [23,24], the leading singularities are invariant under Yangian symmetry [25-28], which is a symmetry combining conformal symmetry and dual conformal symmetry [29-33]. The leading singularity can also be used in constructing one-loop amplitudes by taking this as the rational coefficients of the scalar box integrals. Extending

\footnotetext{
a e-mail: gang.chern@gmail.com
}

this idea to higher-loop amplitudes are reported in [10,3436].

A leading singularity can be viewed as a contour integral over a Grassmannian manifold [37-42]. This expression of the leading singularity keeps many symmetries, in particular, the Yangian symmetry, cyclic and parity symmetries, manifest. On the one hand this new form makes the expression of amplitudes simple and hence easy to calculate. On the other hand it is related to the central ideas in algebraic geometry: Grassmmannian, stratification, algebraic varieties, toric geometry, and intersection theory etc. For leading singularities of the planar amplitudes in $\mathcal{N}=4$ super Yang-Mills (SYM), Arkani-Hamed et al. [43] proposed using positive Grassmannian to study them along with the constructions of the bipartite on-shell - all internal legs are put on shell diagrams [44].

Top forms and the d"log" forms of the Grassmannian integrals are systematically studied for planar diagrams. Each on-shell diagram corresponds to a Yangian invariant, as shown in [31] at tree level and $[32,33,47]$ at loop level. (See also [48,49] for earlier work and [50-64] for a sample of interesting developments thereafter, and [65-77] for a sample of reviews; also a new book has appeared [78].)

We report, in this paper, our detailed and systematic studies of the nonplanar on-shell diagrams which can be decomposed by removing BCFW bridges and applying a $\mathrm{U}(1)$ decoupling relation of the four- and three-point amplitudes (or just decomposable diagrams for convenience). For wide classes of leading singularities, the corresponding on-shell diagrams are decomposable diagrams. We first construct the chain of BCFW decompositions for the on-shell diagrams. During this process we obtain the unglued diagram by cutting an internal line. We prove any unglued diagrams can be categorized into three distinct classes which can be subsequently turned into identity utilizing crucially the permutation relation of generalized Yangian invariants [79]. This construction is presented in Sect. 2. 
We then proceed to the study of the geometry of the leading singularities. We are interested in the constraints encoded in the Grassmannian manifolds and how these constraints determine the integration contours in the top forms. As the cyclic order is destroyed by nonplanarity the integrand of Grassmannian integral also needs to be constructed from scratch. To achieve the above goals we attach nonadjacent BCFW bridges to the planar diagrams and observe how the integrands and the C-matrices transform. Further we can construct the (rational) top form including both integrand and integration contour of any nonplanar leading singularity by attaching (linear) BCFW bridges to the identity diagram in the reverse order of the BCFW decomposition chain from the previous section. This construction is presented in Sect. 3

\section{Scattering amplitudes: BCFW decomposition}

In an on-shell diagram representing an $L$-loop leading singularity, we are free to pull out a planar sub-diagram (unglued diagram) between two internal loop lines - both are also onshell as shown in Fig. 1. ${ }^{1}$ Locally the sub-diagram is planar except that we cannot perform BCFW integrations on these two loop lines. We proved that every such sub-diagram, upon the removal of all BCFW bridges in the permutations, can be cast into one of the three distinct types of skeleton graphs. A $U$ (1)-decoupling relation can be further performed on the latter two types. And the $L$ th loop is unfolded. Unfolding the loops recursively, we obtain the BCFW decomposition chain for the leading singularities of any $L$-loop nonplanar amplitudes. In other words the BCFW chain captures all the information of the leading singularities of the $L$-loop nonplanar graphs. We are thus able to reconstruct the onshell diagrams by attaching BCFW bridges from the identity.

In this section, we will introduce a systematic way of finding the BCFW bridge decomposition chain from the marked permutations $^{2}$ of the unglued diagrams.

\subsection{From permutations to BCFW decompositions}

In this subsection, we derive the BCFW bridge decomposition chain from the permutation of the unglued diagram. First we perform BCFW bridge decompositions on the unglued diagram according to a marked permutation, leaving the cut

\footnotetext{
${ }^{1}$ In this paper we assume there are more than two external lines for this planar sub-diagram. If there is only one external line in the subdiagram, we need to use another method, which is presented in our following paper.

2 Marked permutations refer to permutations with two end points treated specially - the two points are allowed to be marked to themselves or to each other.
}

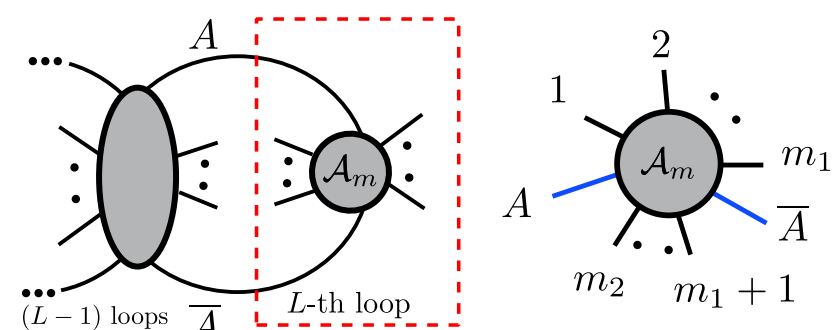

Fig. 1 The $L$-loop's d $l o g$ form can be obtained by reducing to the $(L-1)$-loop problem
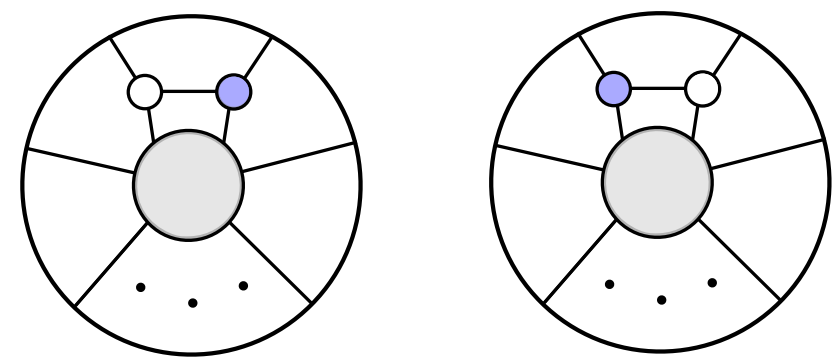

Fig. 2 White-black bridges and black-white bridges

lines untouched. Upon the removal of all adjacent bridges, we will arrive at three categories of skeleton diagrams, noticing that the category type is invariant under the BCFW bridge decomposition. Next for each category of skeleton diagram we construct a specific recipe to decompose it to identity.

From unglued diagram to skeleton diagram All unglued diagrams can be categorized into three groups depending on the permutations of the two cut lines, denoted $A$ and $\bar{A}$,

(1) $\sigma_{1}(A) \neq \bar{A} \& \& \sigma_{1}(\bar{A}) \neq A$;

(2) $\sigma_{2}^{a}(A)=\bar{A} \& \& \sigma_{2}^{a}(\bar{A}) \neq A$, or $\sigma_{2}^{b}(\bar{A})=A \& \& \sigma_{2}^{b}(A)$ $\neq \bar{A}$

(3) $\sigma_{3}(A)=\bar{A} \& \& \sigma_{3}(\bar{A})=A$.

To decompose an unglued diagram, the first step is the full removal of two types of adjacent bridges on the target diagram: the white-black bridge and the black-white bridge as shown in Fig. 2. The changes to the permutation after removing either of them are, respectively, $\sigma \rightarrow \sigma^{\prime}=Z_{2}(k, k+1) \cdot \sigma$ and $\sigma \rightarrow \sigma^{\prime}=Z_{2}\left(\sigma^{-1}(k), \sigma^{-1}(k+1)\right) \cdot \sigma$, where $Z_{2}(k, k+1)$ is a $Z_{2}$ permutation between line $k$ and $k+1$ [43]. For an unglued diagram arisen from a nonplanar leading singularity, the cut line should not be involved in BCFW bridge decompositions as the pair of marked lines are to be glued back eventually. Thus we should restrict the set of allowed BCFW bridge decompositions to those preserving the two marked legs. By this restriction, the group our target unglued diagram originally belongs to will not alter during bridge decompositions. 


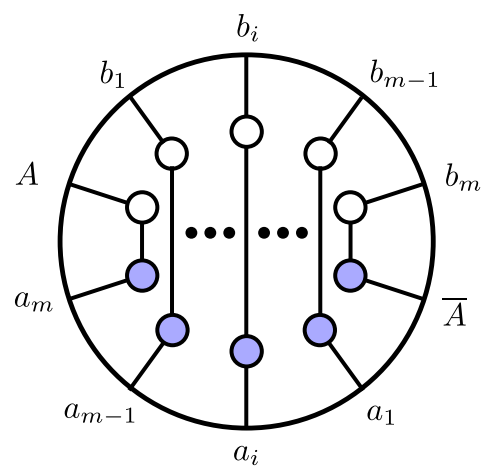

(a) External line Pair

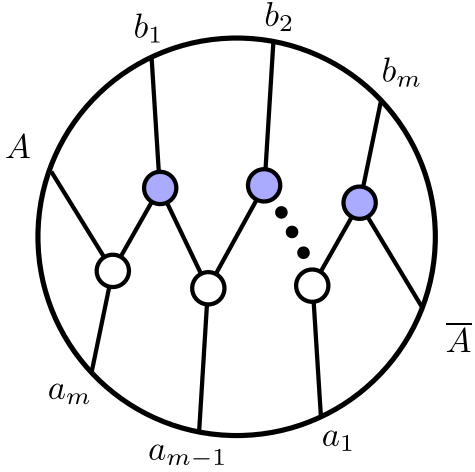

(b) Black-White Chain

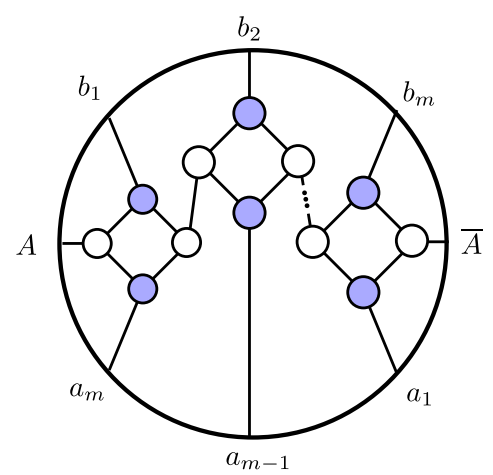

(c) Box Chain

Fig. 3 Skeleton diagrams
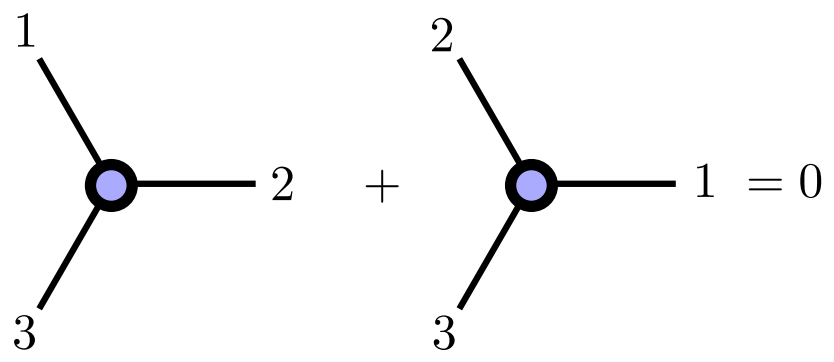

Fig. $4 U(1)$ decoupling relation of 3-point amplitudes

Due to the existence of nonadjacent bridges, an unglued diagram cannot be fully decomposed and will pause at a certain diagram. It is easy to see that after removing all BW and WB bridges the three groups of unglued diagrams will fall into External line pair, Black-White Chain and Box Chain respectively. The three categories are named after their general patterns as shown in Fig. 3. We refer to any diagram belonging to the above three categories as the "skeleton diagram", naming after its skinny looks. As long as we can fully decompose all three skeleton diagrams, it is then direct to obtain the complete decomposition chain of any unglued diagram.

\section{From skeleton diagram to identity}

- External line pair: Most external lines are paired. The external lines next to the internal cut line may also attach to the black/white vertices or be paired with the internal cut line as shown in Fig. 3a. For this type of on-shell diagrams, gluing back the internal lines and removing all pairs will lead to the identity.

- Black-white chain: In this case, white and black vertices are connected together recursively, as shown in Fig. 3b. To further decompose, we use the amplitudes relation $\mathcal{A}\left(a_{1}, a_{2}, a_{3}\right)=-\mathcal{A}\left(a_{1}, a_{3}, a_{2}\right)$ (see Fig. 4) to twist one down-leg to up-leg. Then an adjacent bridge will appear. By removing the new appeared BCFW bridge, the diagram is unfolded into a planar diagram. The diagram can then be decomposed to identity according to its permutation [43].

- Box chain: In this case, the diagram is composed of boxes linked to a chain, as shown in Fig. 3c. Using the $U(1)$ decoupling relation [80] of the four point amplitudes (see Fig. 5), this diagram turns into the sum of two diagrams with adjacent BCFW bridges. The nonadjacent legs of the box will become adjacent under this operation. Performing adjacent BCFW decompositions on both diagrams will unfold the loop and arrive at two planar diagrams, which can be decomposed to identity.

\section{Scattering amplitudes: the top form}

Through the BCFW bridge decompositions we obtain the $\mathrm{d} l o g$ form characterized by the bridge parameters. The $\mathrm{d} l o g$ form can be viewed as an explicit parameterization of a more general integration over the Grassmannian manifold, which is invariant under the $G L(k)$ transformations. The invariant form, known as the "top form," for planar diagrams has been constructed in [43]. In this section, we construct the top form for the nonplanar leading singularities. Recent progress on nonplanar on-shell diagrams can be found in $[45,46]$.

For planar diagrams, the top form manifests the Yangian symmetry: the leading singularities can be written as multidimensional residues in the Grassmannian manifold $\mathcal{G}(k, n)$,

$$
\begin{aligned}
\mathcal{T}_{n}^{k}= & \oint_{C \subset \Gamma} \frac{d^{k \times n} C}{\operatorname{vol}(G L(k))} \frac{\delta^{k \times 4}(C \cdot \tilde{\eta})}{f(C)} \\
& \times \delta^{k \times 2}(C \cdot \tilde{\lambda}) \delta^{2 \times(n-k)}\left(\lambda \cdot C_{\perp}^{\top}\right),
\end{aligned}
$$

where $\Gamma$ is a sub-manifold of $\mathcal{G}(k, n) . \Gamma$ is constrained by a set of linear relations among the columns of $C$ - certain minors of $C$ are to be zero. As any function of the minors of $C, f(C)$, has the scaling property $f(t C)=t^{k \times n} f(C)$. 
Fig. $5 U(1)$ decoupling relation of 4-point amplitudes
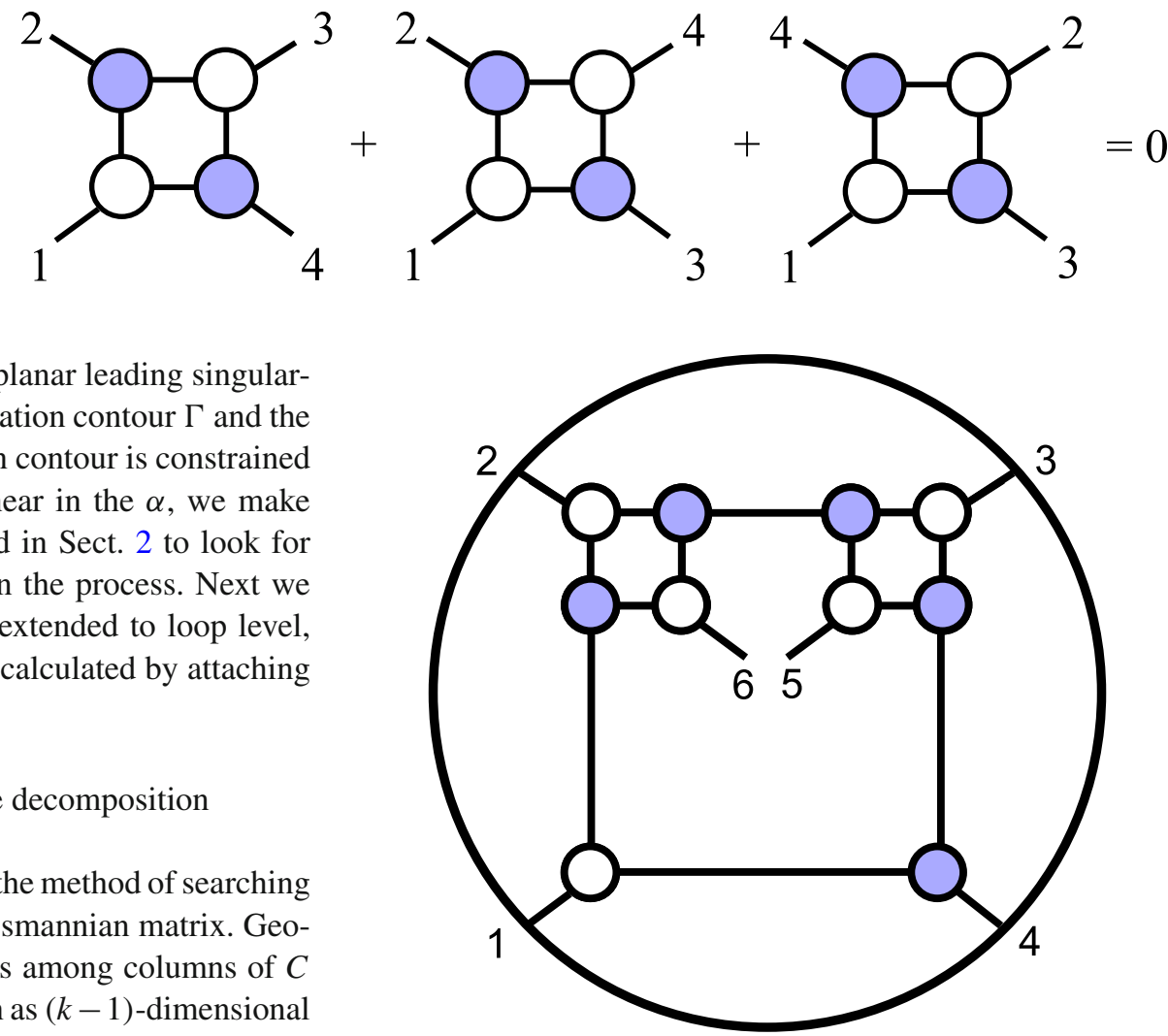

Fig. 6 The BCFW bridge to open the loop of the diagram external line can be map to a point in the projective space. Each time we attach a bridge a constraint will be fixed and the geometry constraints change accordingly. In the Grassmannian matrix, adding a white-black bridge on external lines $\mathbf{a}$, b yields a linear transformations of the two columns, a and $\mathbf{b}, \mathbf{a} \longrightarrow \widehat{\mathbf{a}}=\alpha \mathbf{a}+\mathbf{b}$; whereas adding a black-white bridge means $\mathbf{b} \longrightarrow \widehat{\mathbf{b}}=\alpha \mathbf{b}+\mathbf{a}$.

For convenience, we divide the geometric constraint into two types: simple coplanar constraint and tangled coplanar constraint. Simple coplanarity is just the coplanarity among the points corresponding to the external line. For the tangled coplanar constraint, at least one point is formed by the intersection of super-planes characterized by the point of the external line. We first present an example for each case.

Example for simple coplanarityAs an explicit example, we work out $\mathcal{A}_{6}^{3}$ 's geometry shown in Fig. 6. This diagram becomes planar upon removal of a white-black bridge $(1,4)$. The remaining BCFW bridge decomposition is

$$
\begin{aligned}
& (1,2) \rightarrow(2,3) \rightarrow(3,4) \\
& \quad \rightarrow(2,3) \rightarrow(1,2) \rightarrow(3,5) \rightarrow(2,6) .
\end{aligned}
$$

The linear relations in identity are then

$$
(4)^{0},(5)^{0},(6)^{0} \text {. }
$$

In the Grassmannian matrix, all elements in the three columns are zero. We then reconstruct the diagram through attaching BCFW bridges.
There are eight bridges needed to construct the nonplanar diagram. Each step will diminish one coplanar relation. For instance, the first step is adding a white-black bridge on external line 2 and 6 , leaving column 6 to become $c_{6}+\alpha c_{2}$. The relation $(6)^{0}$ then becomes $(2,6)^{1}$. Similarly upon attaching bridges $(3,5),(1,2),(2,3)$, the coplanar constraints are $(4)^{0},(2,3,5)^{2},(1,2,6)^{2}$. Upon attaching bridge $(3,4)$, constraint $(4)^{0}$ becomes $(3,4)^{1}$. This means that points 3 and 4 merge to a single point. Then the constraint $(2,3,5)^{2}$ can be written as $\left(2,(3,4)^{1}, 5\right)^{2}$. Attaching the bridges consecutively as shown in Table 1, we can finally get the coplanar constraint $(1,2,6)^{2}$ for the on-shell diagram.

Example for tangled coplanarity We consider a nonplanar two-loop diagram, $\mathcal{A}_{6}^{3}$, as shown in Fig. 7.

The BCFW decomposition chain is

$$
\begin{aligned}
& (1,3) \rightarrow(3,5) \rightarrow(2,3) \rightarrow(1,2) \\
& \quad \rightarrow(3,4) \rightarrow(2,3) \rightarrow(3,5) \rightarrow(3,6) .
\end{aligned}
$$

Then the top form of the diagram can be reconstructed by attaching these bridges one by one. There are eight bridges and each one diminish a coplanar constraint as shown in Table 2.

The first seven bridges attached yield simple coplanar constraints. Then the geometry constraints are $(2,3,4)^{2}$, $(3,5,6)^{2}$, which indicates that points $2,3,4$ and 3, 5, 6 are 
Table 1 The evolution of the geometry constraints under adding BCFW bridges. The first row is the linear relation in the identity diagram and the column on the left represents the bridge decomposition chain

\begin{tabular}{ll}
\hline Bridge & Coplanar constraints \\
\hline Begin & $(4)^{0}(5)^{0}(6)^{0}$ \\
$(2,6)$ & $(4)^{0}(5)^{0}(2,6)^{1}$ \\
$(3,5)$ & $(4)^{0}(3,5)^{1}(2,6)^{1}$ \\
$(1,2)$ & $(4)^{0}(3,5)^{1}(1,2,6)^{2}$ \\
$(2,3)$ & $(4)^{0}(2,3,5)^{2}(1,2,6)^{2}$ \\
$(3,4)$ & $(3,4)^{1}\left(2,(3,4)^{1}, 5\right)^{2}(1,2,6)^{2}$ \\
$(2,3)$ & $(2,3,4,5)^{2}(1,2,6)^{2}$ \\
$(1,2)$ & $(3,4,5)^{2}(1,2,6)^{2}$ \\
$(1,4)$ & $(1,2,6)^{2}$ \\
\hline
\end{tabular}

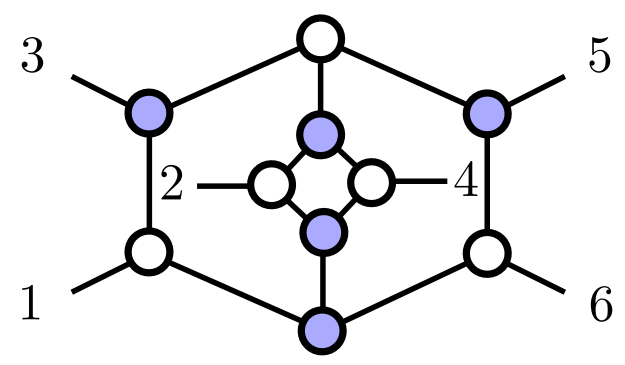

Fig. 7 An example of a nonplanar two-loop diagram $\mathcal{A}_{6}^{3}$

Table 2 The evolution of the geometry constraints under adding BCFW bridges

\begin{tabular}{ll}
\hline Bridge & Coplanar constraints \\
\hline Begin & $(4)^{0}(5)^{0}(6)^{0}$ \\
$(3,6)$ & $(4)^{0}(5)^{0}(3,6)^{1}$ \\
$(3,5)$ & $(4)^{0}(3,5)^{1}(5,6)^{1}$ \\
$(2,3)$ & $(4)^{0}(2,3,5)^{2}(5,6)^{1}$ \\
$(3,4)$ & $(34)^{1}(2,3,5)^{2}(5,6)^{1}$ \\
$(1,2)$ & $(3,4)^{1}(5,6)^{1}$ \\
$(2,3)$ & $(2,3,4)^{2}(5,6)^{1}$ \\
$(3,5)$ & $(2,3,4)^{2}(3,5,6)^{2}$ \\
$(1,3)$ & $\frac{(234)}{(214)}-\frac{(356)}{(156)}=0$ \\
\hline
\end{tabular}

collinear, respectively, as shown in Fig. 8. Then we attach the last bridge. As discussed above, point 3 is shifted to $\hat{3}$ along the line-(31). It seems the $2, \hat{3}, 4$ and $\hat{3}, 5,6$ are not collinear anymore and the two constraints are removed together. In fact, according to Fig. 8, there is another coplanar constraint that is the intersect point $(1 \widehat{3}) \cap(24)$ lie in the line of $(56)$. For convenience, we denote this tangled coplanar relation as $((1 \widehat{3}) \cap(24) 56)$. The geometry evolution under the last bridge $(1,3)$ is shown in Fig. 8 .

General simple coplanar constraints We first discuss the cases without tangled coplanar constraints. We can classify

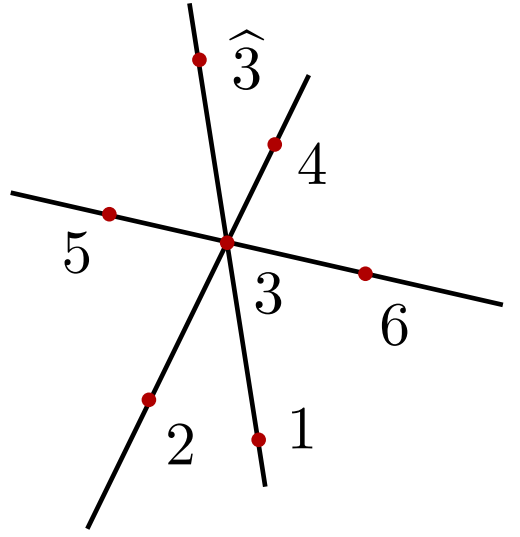

Fig. 8 The constraint of the diagram is $((1 \widehat{3}) \cap(24) 56)$. In projective space, it means line $(1 \widehat{3})$, line $(24)$ and line $(56)$ all intersect on point 3 . Such a constraint can only appear in multi-loop nonplanar on-shell diagrams

the coplanar constraints into four sets according to the elements:

1. $\left(\mathbf{a}, \mathbf{b}, 1,2 \ldots m_{1}\right)^{k_{1}}$

2. $\left(\mathbf{a}, 1,2 \ldots m_{2}\right)^{k_{2}}$

3. $\left(\mathbf{b}, 1,2 \ldots m_{3}\right)^{k_{3}}$

4. $\left(1,2, \ldots m_{4}\right)^{k_{4}}$

where $k_{1}$ to $k_{4}$ are the ranks of the minors. Without loss of generality, we shall make $k_{1}<m_{1}+2, k_{2}<m_{2}+1$, $k_{3}<m_{3}+1$ and $k_{4}<m_{4}$. We call the minor complete if only if adding any other column to the matrix will make the rank increase by one. From now on we shall assume that all the minors in the above sets are complete in the following discussion. In fact, incomplete minors can always be transformed into the complete ones by adding to the bracket all the necessary elements while keeping the rank unaltered.

Attaching a white-black bridge does not change the rank of minors in set 1 since $a$ and $b$ are both in this set. The minors in set 2 and set 4 remain unaltered since $b$ is excluded from these two sets. $(\alpha \mathbf{a}+\mathbf{b}) \notin \operatorname{span}\left\{\mathbf{b}, \mathbf{1}, \mathbf{2} \ldots \mathbf{m}_{\mathbf{3}}\right\}$, thus after adding a white-black bridge the only set with its rank altered is set 3 . The minors in set 3 can generate two new linear relations: $\left(a, b, 1,2 \ldots m_{3}\right)^{k_{3}+1}$ and $\left(1,2 \ldots m_{3}\right)^{k_{3}}$. Similarly, upon attaching a black-white bridge, the minors in set 2 will become $\left(a, b, 1,2 \ldots m_{2}\right)^{k_{2}+1}$ and $\left(1,2 \ldots m_{2}\right)^{k_{2}}$. We have completed the discussion of how constraints alter during each step of bridge decompositions.

Next we turn to attaching bridges starting from the identity with the identity diagram being a matrix with $n-k$ columns of zero vectors. Each time we attach a BCFW bridge, the number of independent geometric constraints will decrease by one. This can be proved through the following procedure. Attaching the bridge $(a, b)$ affects the linear relation involving $\mathbf{b}$. The only exceptions are the relations containing both $a$ and $b$, which will not be affected by the bridge $(a, b)$, 

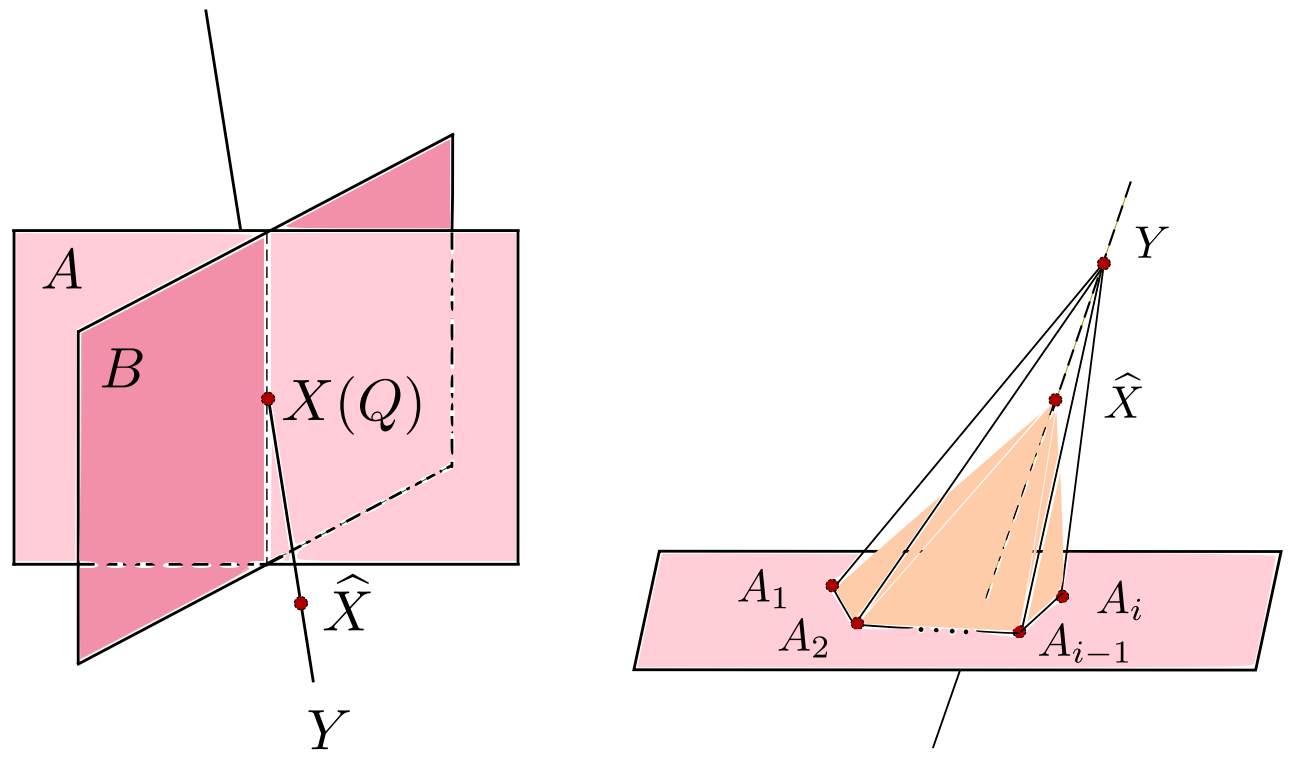

Fig. 9 The diagrammatic notation of Eq. 4. The plane or hyperplane $A=\operatorname{Span}\left(X A_{1} A_{2} \ldots A_{i}\right)$ and $B=\operatorname{Span}\left(X B_{1} B_{2} \ldots B_{i}\right) . \alpha$ can be regarded as the ratio of two hyperpolyhedron's volumes

$(b, 1,2 \ldots m)^{k} \longrightarrow\left\{\begin{array}{l}\left(\mathbf{a}, \mathbf{b},(1,2 \ldots \mathbf{m})^{k}\right)^{k+1} \\ (1,2 \ldots m)^{k}\end{array}\right.$

If $k=m$, the linear relation $(1,2 \ldots m)^{k}$ does not give rise to any constraint, thus $(a, b, 1,2 \ldots m)^{k+1}$ has one higher rank than $(b, 1,2 \ldots m)^{k}$. The constraints' number is then diminished by one upon attaching the bridge. If $k<m$, the coplanar constraints $(b, 1,2 \ldots m)^{k}$ can be decomposed to $\left(b,(1,2 \ldots m)^{k}\right)^{k}$ and $(1,2 \ldots m)^{k}$. The independent constraints after attaching the bridge are $\left(a, b,(1,2 \ldots m)^{k}\right)^{k+1}$ and $(1,2 \ldots m)^{k}$. Comparing the constraints between $\left(b,(1,2 \ldots m)^{k}\right)^{k}$ and $\left(a, b,(1,2 \ldots m)^{k}\right)^{k+1}$, the number of constraints is reduced by one upon adding the BCFW bridge.

General tangled coplanar constraints Now we discuss the tangled coplanar constraints. When we attach a BCFW bridge, $X \rightarrow \widehat{X}=X+\alpha Y{ }^{3}$ There are two constraints $\left(X A_{1} A_{2} \ldots A_{i_{1}}\right)^{i_{1}}$ and $\left(X B_{1} B_{2} \ldots B_{i_{2}}\right)^{i_{2}}$ both containing the shifting leg. Without losing generality, we assume that there is no constraints among $A_{1}, A_{2} \ldots A_{i_{1}}$ and $B_{1}, B_{2} \ldots B_{i_{2}}$. Upon attaching a $\mathrm{BCFW}$ bridge a tangled constraint could be obtained:

$\alpha=\frac{V^{i_{1}+1}\left(\widehat{X} A_{1} \ldots A_{i_{1}}\right)}{V^{i_{1}+1}\left(Y A_{1} \ldots A_{i_{1}}\right)}=\frac{V^{i_{2}+1}\left(\widehat{X} B_{1} \ldots B_{i_{2}}\right)}{V^{i_{2}+1}\left(Y B_{1} \ldots B_{i_{2}}\right)}$,

where $V$ stands for the volume of the hyperpolyhedron.

\footnotetext{
3 The bridge at least shifts one of the constraints linearly. We will show in next section that this condition is equivalent to that the top form is rational.
}

It is indeed a geometric constraint on the Grassmannian manifold - the point of intersection of one line and one hyperplane lying on another hyperplane:

$\left((\widehat{X} Y) \cap\left(B_{1} B_{2} \ldots B_{i_{2}}\right) A_{1} A_{2} \ldots A_{i_{1}}\right)$.

We denote the intersection point of $(\widehat{X} Y) \cap\left(B_{1} B_{2} \ldots B_{i}\right)$ as $Q=C_{1} \widehat{X}+C_{2} Y$. Since $Q$ also lies in the plane $\left(B_{1} B_{2} \ldots B_{i}\right)$,

$C_{1} V^{i_{2}+1}\left(\widehat{X} B_{1} B_{2} \ldots B_{i_{2}}\right)+C_{2} V^{i_{2}+1}\left(Y B_{1} B_{2} \ldots B_{i_{2}}\right)=0$.

Thus the initial constraint $\left(Q A_{1} A_{2} \ldots A_{i_{1}}\right)^{i_{1}}$ directly yields Eq. 3. One may have noticed that the point $Q$ is precisely the point $X$ before shifting. If we go on attaching another bridge that involves this tangled constraint, the set of constraints can again be written as minors of the Grassmannian matrix (Fig. 9).

Therefore we conclude that general constraints can always be labeled using nested spans and intersections. Consider attaching a linear BCFW bridge $(Y, X)$ in an arbitrary amplitude, a constraint to be shifted is

$$
\begin{aligned}
M(X) \equiv & \left(\cdots ( X A _ { 1 } ^ { ( 0 ) } A _ { 2 } ^ { ( 0 ) } \cdots A _ { a _ { 0 } } ^ { ( 0 ) } ) \cap \left(B_{1}^{(1)} B_{2}^{(1)}\right.\right. \\
& \left.\left.\cdots B_{b_{1}}^{(1)}\right) A_{1}^{(1)} A_{2}^{(1)} \cdots A_{a_{1}}^{(1)}\right) \\
& \left.\cap\left(B_{1}^{(2)} B_{2}^{(2)} \cdots B_{b_{2}}^{(2)}\right) A_{1}^{(2)} A_{1}^{(2)} \cdots A_{a_{2}}^{(2)}\right) \\
& \left.\cdots \cap\left(B_{1}^{(m)} B_{2}^{(m)} \cdots B_{b_{m}}^{(m)}\right) A_{1}^{(m)} A_{2}^{(m)} \cdots A_{a_{m}}^{(m)}\right)
\end{aligned}
$$

with $X$ being the external line to be shifted, $A^{(\cdot)}$ and $B^{(\cdot)}$ denoting two sets of external lines. If column $X \in \operatorname{Set}\left[A^{(\cdot)}\right]$ or $X \in \operatorname{Set}\left[B^{(\cdot)}\right]$, they can be freely replaced by $\hat{X}$ after attaching the bridge involving $X$. Otherwise the constraint will be a nonlinear function of $\alpha$, resulting in an irrational 

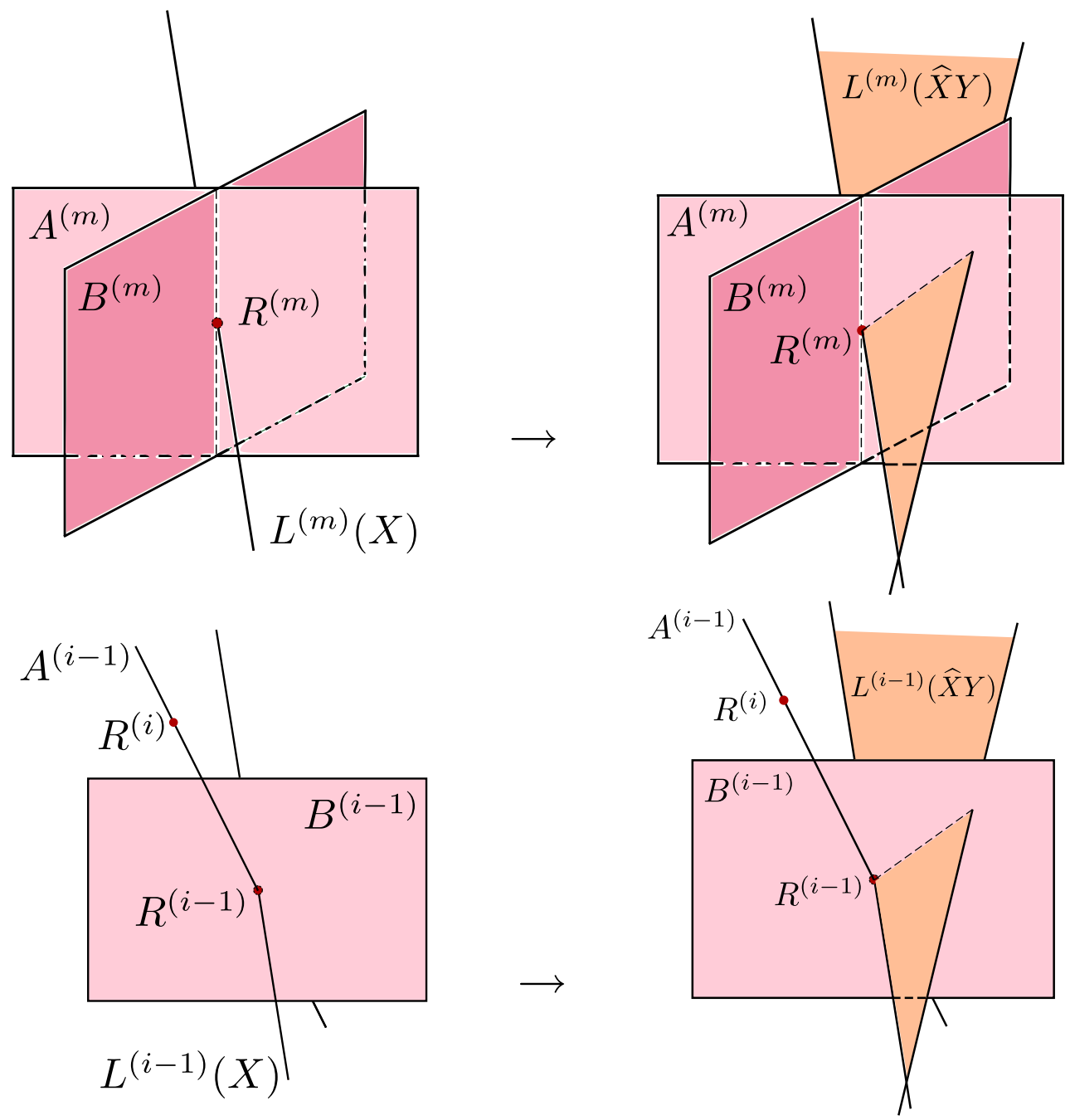

Fig. 10 The geometric relation indicated by the ground level (the upper figures) and the $i$ th level (the lower figures) of the nested minor. The left figures are the relations before the $\mathrm{BCFW}$ shift. The right figures are the relations after the BCFW shift

top form. We present a counter example in the appendix to illustrate this point. We can then simplify $M(X)$ as follows:

$\left(L^{(m)}(X) \cap\left(B_{1}^{(m)} B_{2}^{(m)} \cdots B_{b_{m}}^{(m)}\right) A_{1}^{(m)} \bar{A}_{2}^{(m)} \cdots \bar{A}_{\bar{a}_{m}}^{(m)}\right)$,

where $\bar{A}^{(\cdot)}$ are some points or hyperplanes composed by $A^{(\cdot)}$ and $B^{(\cdot)}$ and are easily obtained through a simple relation,

$$
\begin{aligned}
\left(A_{1} A_{2} A_{3}\right) \cap P & =\left(\left(\left(A_{1} A_{2}\right) \cap P\right)\left(\left(A_{2} A_{3}\right) \cap P\right)\right) \\
& =\left(\left(\left(A_{1} A_{2}\right) \cap P\right) \bar{A}\right) .
\end{aligned}
$$

After the shift the constraint $M(X)$ is removed. In order to obtain the other constraints, we look for the $\widehat{C}$ representation of $X$. This is achieved by unfolding the nested intersections level by level.

To write a constraint in a compact form and make the geometric relations encoded manifest, We define a line $L(X)$, for $i \in[2, m]$
$L^{(i)}(X) \equiv\left(L^{(i-1)}(X) \cap\left(B_{1}^{(i-1)} \cdots B_{b_{i-1}}^{(i-1)}\right) A_{1}^{(i-1)}\right)$.

We further define a point $R^{(i)}(X) \equiv L^{(i)}(X) \cap\left(B_{1}^{(i)} \cdots B_{b_{i}}^{(i)}\right)$, for $i \in[2, m]$. Given a minor $M(X)$, we could obtain the point $R^{(m)}(X)$ as

$R^{(m)}(X)=L^{(m)}(\widehat{X} Y) \cap\left(B_{1}^{(m)} \cdots B_{b_{m}}^{(m)}\right) \cap\left(A_{1}^{(m)} \cdots \bar{A}_{\bar{a}_{m}}^{(m)}\right)$.

All levels of $R^{(i)}(X)$ can be recursively obtained according to

$$
R^{(i-1)}=\left(R^{(i)} A_{1}^{(i-1)}\right) \cap\left(L^{(i-1)}(\widehat{X} Y) \cap\left(B_{1}^{(i-1)} \cdots B_{b_{i-1}}^{(i-1)}\right)\right) .
$$

The geometrical relations is shown in Fig. 10. Finally we are able to denote the column $X$ using the columns in the shifted Grassmannian, $X=\left(R^{(1)} A_{1}^{(0)}\right) \cap(\widehat{X} Y)$. After removing the constraint $M(X)=0$, the remaining constraints are invariant under the $\widehat{C}$ representation of $X$, making them independent of the shift $(Y, X)$. 
For now we have obtained geometry constraints according to BCFW bridge decomposition chain. We would like to stress that our approach can be applied to seeking all loop leading singularity's geometry constraints. During the process, we introduced a method that the constraints are independently and completely represented. The constraints of the graph constructed by any "top form bridge" are immediately obtained using our method. Thus the top form integrations' contour $\Gamma$ is determined.

\subsection{Rational top forms and linear BCFW bridges}

Attaching BCFW bridges and using the 3- or 4-point amplitude relations reductively, all nonplanar diagrams can be constructed and their $d \log$ forms be found. We should stress, however, that not all nonplanar on-shell diagrams have rational top forms; and it is worth to remark on which kind of nonplanar on-shell diagrams can have rational top forms. We address this question by building up an equivalent relation between rational top form and linear BCFW bridges. If a BCFW bridge results in the shifted constraint function to be a linear function of $\alpha$, we call this BCFW bridge a linear $B C F W$ bridge.

A constraint function $F_{i}$ is a rational function of the minors of the Grassmannian matrix, $C$. Altogether they span an algebraic ideal $\mathcal{I}\left[\left\{F_{i}\right\}\right]$. Under a BCFW shift $X \rightarrow \widehat{X}=X+\alpha Y$, a constraint is eliminated, with $C$ being transformed to $\widehat{C}$. The transformed $f^{\prime}(\widehat{C})$ is also rational iff $\alpha$ is also a rational function of $\widehat{C}$.

Next we need to show that rationality of $\alpha$ is guaranteed by the linear BCFW shifts. To prove their equivalence, assuming $\alpha=P(\widehat{C}) / Q(\widehat{C})$, where $P, Q$ are polynomials of minors of $\widehat{C}$. Expanding $P, Q$ as polynomials of $\alpha$ as well as the minors of $C$,

$\alpha=\frac{P_{0}+P_{1} \alpha+P_{2} \alpha^{2}+\cdots+P_{N} \alpha^{N}}{Q_{1}+Q_{2} \alpha+\cdots+Q_{N} \alpha^{N-1}}$.

The coefficient in each power of $\alpha$, such as $P_{0}, P_{1}-Q_{1}, P_{2}-$ $Q_{2}, \cdots, P_{N}-Q_{N}$, is supposed to vanish. If any of them appears to be nonzero, it must fall into the ideal $\mathcal{I}\left[\left\{F_{i}\right\}\right]$. This means that all the coefficients are constraints of $C$. Under the shift, $P_{i}$ and $Q_{i}$ become

$$
\begin{aligned}
& \widehat{P}_{i}=P_{i}+\sum_{j=1}^{N-i}\left(\begin{array}{c}
j \\
i+j
\end{array}\right) P_{i+j} \alpha^{j}, \\
& \widehat{Q}_{i}=Q_{i}+\sum_{j=1}^{N-1-i}\left(\begin{array}{c}
j \\
i+j
\end{array}\right) P_{i+j} \alpha^{j} .
\end{aligned}
$$

The constraint $P_{N}-Q_{N}$ remains the same after the shift $\widehat{P}_{N}-\widehat{Q}_{N}=P_{N}-Q_{N}$. The constraint $P_{N-1}-Q_{N-1}=0$ appears linearly dependent on $\alpha$ upon

$\widehat{P}_{N-1}-\widehat{Q}_{N-1}=\left[N P_{N}-(N-1) Q_{N}\right] \alpha$.
If $\left[N P_{N}-(N-1) Q_{N}\right]$ does not vanish then the constraint $P_{N-1}-Q_{N-1}=0$ is the removed. Otherwise $P_{N}=Q_{N}=$ 0 and the constraint $P_{N-2}-Q_{N-2}=0$ is shifted linearly,

$\widehat{P}_{N-2}-\widehat{Q}_{N-2}=\left[(N-1) P_{N-1}-(N-2) Q_{N-1}\right] \alpha$.

Since $P(\widehat{C})$ cannot be totally independent of $\alpha$, we can trace the constraints from $N$ to 0 until we find one constraint $\left(P_{i}-Q_{i}\right)$, which is a linear function of $\alpha$ after a shift. This constraint is then the constraint being removed.

The proof of the reverse is also straightforward: if one constraint becomes a linear function of $\alpha$ under a shift, for instance

$F_{i}(C) \rightarrow F_{i}(\widehat{C})=F_{i}(C)+F_{i}^{\prime}(C) \alpha=F_{i}^{\prime}(\widehat{C}) \alpha$,

where $F_{i}(C)$ vanishes and $F_{i}^{\prime}(C)$ is invariant under the shift, we have $\alpha=F_{i}(\widehat{C}) / F_{i}^{\prime}(\widehat{C})$. Note that the remaining $s-1$ constraints can be written in the form $F_{i}(\cdots \widehat{X}-\alpha Y \cdots)=$ 0 , for $i \in[2, s]$, which are invariant under the shift.

Finally we conclude that upon adding a BCFW bridge the on-shell diagram resulted has a rational top form if and only if the shift on the algebra ideal $\mathcal{I}$ is linear. For a generic on-shell diagram, BCFW bridges can be added in an arbitrary manner and the transformations on the constraints are complicated. Top forms can be obtained if and only if when the BCFW parameters shift the constraints linearly. This type of bridges is thus called linear $B C F W$ bridges. In the construction of top forms one should avoid using BCFW bridges that shift the constraints in a nonlinear manner.

\subsection{From BCFW decompositions to top forms}

To obtain the top form of scattering amplitudes, besides the geometric constraints, we also need to get the integrand, $f(C)$. It must then contain those poles equivalent to the constraints in $\Gamma$ to keep the non-vanishing of the circleintegration in Eq. 1. Each BCFW bridge removes one pole in $f(C)$ by shifting a zero minor to be nonzero: in tangled cases the poles in the integrand must change their forms accordingly.

To see this we parameterize the constraint matrix, $C$, using the BCFW parameter, $\alpha$. In the last BCFW shift $X \rightarrow \widehat{X}=$ $X+\alpha Y$, several minors in $f(C)$ become functions of $\alpha$. There exists at least one minor $M_{0}(\widehat{X})=M_{0}(X)+\alpha R(Y)$ having a pole at $\alpha=0$. After this shift, $M_{0}(X) \rightarrow M_{0}(\widehat{X})$, the constraint $M_{0}(X)=0$ is removed. And $\alpha=M_{0}(\widehat{X}) / R(Y)$ is then a rational function of $\widehat{C}$ and can be subtracted from the other shifted minors to obtain the shift-invariant minors of $\widehat{C}, M_{i}(X)=M_{i}(\widehat{X}-\alpha Y)$. This is demonstrated in Sect. 3.2 We can further attach a BCFW bridge to the integrand,

$$
f(\widehat{C})=M_{0}(\widehat{X}) \prod_{i} M_{i}(\widehat{X}-\alpha Y) \times\left(\begin{array}{c}
\text { minors } \\
\text { without } \alpha
\end{array}\right) .
$$




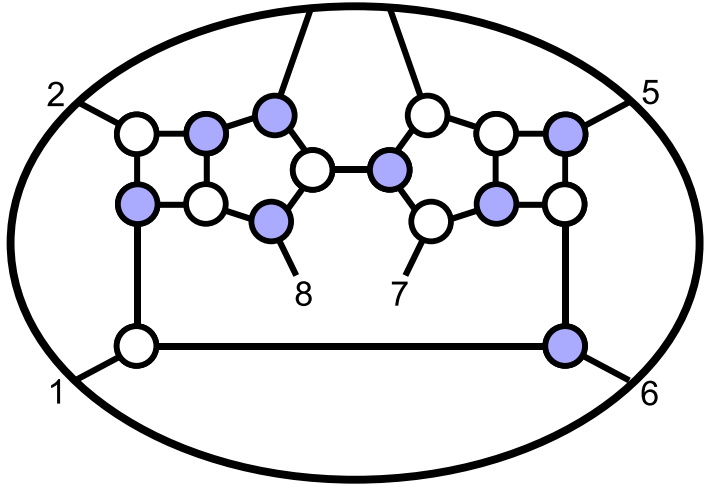

Fig. 11 A nonplanar on-shell diagram of $\mathcal{A}_{8}^{4}$

In this way top forms of leading singularities of scattering amplitudes can thus be obtained - be it planar or nonplanar - and from tree level to all loops. We illustrate our method below with several examples: searching for the constraints and calculating the top form integrand.

\subsection{Several examples}

A one-loop example of attaching a nonadjacent bridge As an application, we take the nonplanar diagram in Fig. 11 as an example.

$\widehat{\mathcal{T}_{8}^{4}}=\oint_{\widehat{\Gamma}} \frac{d^{4 \times 8} \widehat{C}}{\operatorname{vol}(G L(4))} \frac{(1345)}{(1234)(2345)(3456)(3457)(1456)(3458}$
According to the permutation of the planar diagrams
before attaching the bridge $(1,6)$, linear relations of the dia-
gram are $(4,5,6,7)^{2}$ and $(8,1,2,3)^{3}$. And the top form is
$\mathcal{T}_{8}^{4}=\oint_{\Gamma} \frac{d^{16} C}{(1234)(2345)(3456)(4567)(5678)(6781)(7812)(8123)}$,

where we have omitted the delta functions for clarity. Attaching bridge $(1,6)$, the coplanar constraint $(8,1,2,3)^{3}$ is unaffected while $(4,5,6,7)^{2} \rightarrow(4,5,7)^{2},\left((4,5,7)^{2} 1 \hat{6}\right)^{3}$, where $\hat{\sigma}=6+\alpha 1$. We choose the shifted pole as (3456). The shift parameter $\alpha$ can be written as $\alpha=\frac{(345 \hat{6})}{(3451)}$. According to Eq. 6, we get

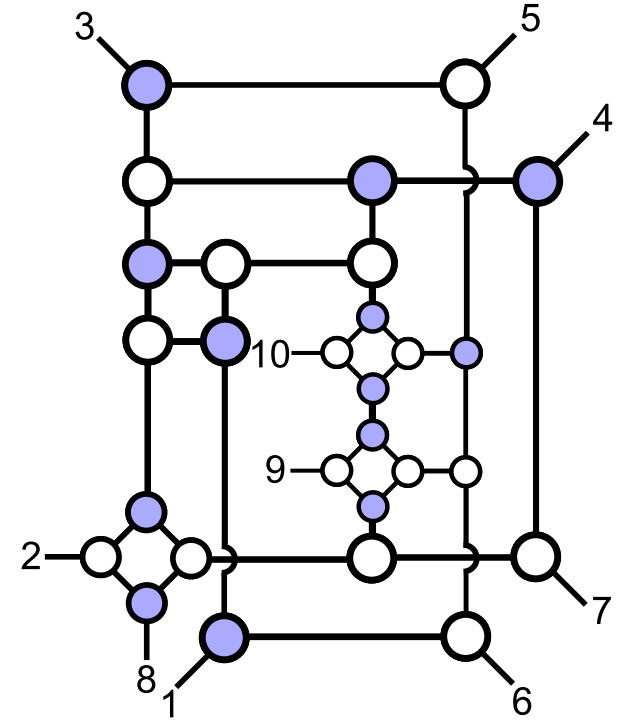

Fig. 12 A nonplanar diagram of $\mathcal{A}_{10}^{4}$

and

$(5(\hat{\sigma}-\alpha 1) 78)=(5 \hat{6} 78)-\frac{(345 \hat{6})}{(3451)}(5178)=-\frac{(3458)(1567)}{(3451)}$.

Finally we obtain the top form of $\mathcal{A}_{8}^{4}$ :

$\mathcal{T}_{8}^{4}=\oint_{\Gamma} \frac{d^{16} C}{(1234)(2345)(3456)(45(\hat{b}-\alpha 1) 7)(5(\hat{b}-\alpha 1) 78)(6781)(7812)(8123)}$,

where

$$
(45(\hat{\sigma}-\alpha 1) 7)=(45 \hat{6} 7)-\frac{(345 \hat{6})}{(3451)}(4517)=-\frac{(3457)(1456)}{(3451)}
$$

Therefore, we can always construct the top form of nonplanar diagrams by attaching adjacent and nonadjacent bridges on the identity diagram step by step.

A tangled two-loop example At multi-loop level the geometric constraints for a nonplanar leading singularity can be highly tangled, as the diagrams cannot, in general, be reduced to the planar ones by KK relation [80].

The diagram is a planar one before attaching the bridge $(3,5)$ in the seventh step and the top form is

$\mathcal{T}_{6}^{3}=\oint_{\Gamma} \frac{d^{9} C}{(123)(234)(345)(456)(561)(612)}$, where we have omitted the delta functions for clarity. After attaching BCFW bridge $(3,5)$, the on-shell diagram become planar. The top form can be obtained directly as in the previous paragraph, 
Table 3 The evolution of the geometric constraints with adding BCFW bridges for the diagram in Fig. 12

\begin{tabular}{|c|c|c|c|c|c|c|}
\hline & $(3)^{0}$ & $(4)^{0}$ & $(7)^{0}$ & $(8)^{0}$ & $(9)^{0}$ & $(10)^{0}$ \\
\hline$(6,10)$ & $(3)^{0}$ & $(4)^{0}$ & $(7)^{0}$ & $(8)^{0}$ & $(9)^{0}$ & $(6,10)^{1}$ \\
\hline$(2,6)$ & $(3)^{0}$ & $(4)^{0}$ & $(7)^{0}$ & $(8)^{0}$ & $(9)^{0}$ & $(2,6,10)^{2}$ \\
\hline$(6,9)$ & $(3)^{0}$ & $(4)^{0}$ & $(7)^{0}$ & $(8)^{0}$ & $(6,9)^{1}$ & $(2,6,10)^{2}$ \\
\hline$(5,6)$ & $(3)^{0}$ & $(4)^{0}$ & $(7)^{0}$ & $(8)^{0}$ & $(5,6,9)^{2}$ & $(2,6,10)^{2}$ \\
\hline$(6,9)$ & $(3)^{0}$ & $(4)^{0}$ & $(7)^{0}$ & $(8)^{0}$ & $(5,6,9)^{2}$ & $(2,6,9,10)^{3}$ \\
\hline$(6,8)$ & $(3)^{0}$ & $(4)^{0}$ & $(7)^{0}$ & $(6,8)^{1}$ & $(5,6,9)^{2}$ & $(2,6,9,10)^{3}$ \\
\hline$(6,7)$ & $(3)^{0}$ & $(4)^{0}$ & $(6,7)^{1}$ & $(6,8)^{1}$ & $(5,6,9)^{2}$ & $(2,6,9,10)^{3}$ \\
\hline$(5,6)$ & $(3)^{0}$ & $(4)^{0}$ & $(7,8)^{1}$ & $(5,6,8)^{2}$ & $(5,8,9)^{2}$ & $(2,8,9,10)^{3}$ \\
\hline$(2,5)$ & $(3)^{0}$ & $(4)^{0}$ & $(7,8)^{1}$ & $(6,8,9)^{2}$ & $(2,5,8,9)^{3}$ & $(2,8,9,10)^{3}$ \\
\hline$(2,4)$ & $(3)^{0}$ & $(2,4)^{1}$ & $(7,8)^{1}$ & $(6,8,9)^{2}$ & $(2,5,8,9)^{3}$ & $(2,8,9,10)^{3}$ \\
\hline$(1,2)$ & $(3)^{0}$ & $(1,2,4)^{2}$ & $(7,8)^{1}$ & $(6,8,9)^{2}$ & $(4,5,8,9)^{3}$ & $(4,8,9,10)^{3}$ \\
\hline$(2,3)$ & $(2,3)^{1}$ & $(1,2,4)^{2}$ & $(7,8)^{1}$ & $(6,8,9)^{2}$ & $(4,5,8,9)^{3}$ & $(4,8,9,10)^{3}$ \\
\hline$(1,2)$ & $(1,2,3)^{2}$ & $(1,3,4)^{2}$ & $(7,8)^{1}$ & $(6,8,9)^{2}$ & $(4,5,8,9)^{3}$ & $(4,8,9,10)^{3}$ \\
\hline$(8,2)$ & $(1,2,3,8)^{3}$ & $(1,3,4)^{2}$ & $(7,8)^{1}$ & $(6,8,9)^{2}$ & $(4,5,8,9)^{3}$ & $(4,8,9,10)^{3}$ \\
\hline$(2,8)$ & $(1,2,3,8)^{3}$ & $(1,3,4)^{2}$ & $(2,7,8)^{2}$ & $(6,7,9)^{2}$ & $(4,5,7,9)^{3}$ & $(4,7,9,10)^{3}$ \\
\hline$(6,1)$ & $(2,3,4,8)^{3}$ & $(1,3,4,6)^{3}$ & $(2,7,8)^{2}$ & $(6,7,9)^{2}$ & $(4,5,7,9)^{3}$ & $(4,7,9,10)^{3}$ \\
\hline$(3,4)$ & $(2,3,4,8)^{3}$ & $(1,3,4,6)^{3}$ & $(2,7,8)^{2}$ & $(6,7,9)^{2}$ & $(5,7,9,10)^{3}$ & \\
\hline$(5,3)$ & & $((5,3) \cap(2,4,8), 1,4,6)^{3}$ & $(2,7,8)^{2}$ & $(6,7,9)^{2}$ & $(5,7,9,10)^{3}$ & \\
\hline$(7,4)$ & & & $(2,7,8)^{2}$ & $(6,7,9)^{2}$ & $(5,7,9,10)^{3}$ & \\
\hline
\end{tabular}

$\mathcal{T}_{6}^{3}=\oint_{\Gamma} \frac{d^{9} C(361)}{(123)(234)(345)(356)(146)(561)(612)}$

here the constraints $\Gamma$ are determined by the linear relation $(2,3,4)^{2}$ and $(3,5,6)^{2}$ as shown in Table 2 . We do not distinguish the labels $\Gamma$ for each step in adding the BCFW bridges. We work according to the discussion above, upon attaching the last BCFW bridge $(1,3)$. Its exact expression can be obtained by transforming the constraints in the last step linearly as $(234)=(356)=0 \rightarrow(234)=0, \frac{(234)}{(214)}-\frac{(356)}{(156)}=0$.

Adding the bridge $(1,3)$ and the elimination of $(234)=0$ leaves $\frac{(234)}{(214)}-\frac{(356)}{(156)}=0$ invariant. Applying the same linear transformation to the denominator:

$\frac{1}{(234)(356)} \rightarrow \frac{-1}{(2 \widehat{3} 4)\left(\frac{-(156)(2 \widehat{3} 4)}{(214)}+(\widehat{356})\right)}$,

we extract the top form
MHV top form and its simplification The MHV top form can be further simplified. We can always transform any nonplanar MHV top form into a summation of several top forms. These generated top forms share features that their numerators of the integrands equal 1 and the minors in $f(C)$ are of cyclic orders, which are exactly those of planar MHV top forms. This yields a strong proof that any nonplanar MHV amplitude is a summation of several planar amplitudes.

To see this, let us consider the top form of $\mathcal{A}_{n}^{2}$. Attaching a nonadjacent bridge $(a, b)$ to a planar diagram yields

$\widehat{\mathcal{T}_{n}^{2}}=\int \frac{d \alpha}{\alpha} \oint_{\Gamma} \frac{d^{2 \times n} C}{\operatorname{vol}(G L(2))} \frac{\delta(C)}{(12) \cdots(b-1, b)(b, b+1) \cdots(n 1)}$

Without losing generality, we assume the pole at $(b-1, b)=$ 0. Following the same procedure illustrated in Eq. 1, we obtain

$$
\widehat{\mathcal{T}_{6}^{3}}=\oint_{\Gamma} \frac{d^{9} \widehat{C}(214)^{2}(\widehat{3} 61)}{(12 \widehat{3})(2 \widehat{3} 4)((1 \widehat{3}) \cap(24) 56)((1 \widehat{3}) \cap(24) 45)(146)(561)(612)} .
$$

$$
\frac{1}{f(\widehat{C})}=\frac{(a, b-1)}{(12) \cdots(b-2, b-1)(b-1, \widehat{b})(a, \widehat{b})(b+1, b-1)(b+1, b+2) \cdots(n 1)} .
$$


Since $a<b-1$, we define $a+m=b-1\left(m \in \mathbb{Z}^{+}\right)$.

- If $m=1$, the numerator is then $(b-2, b-1)$ and the integrand can be simplified to a term with its numerator equaling one and $f(C)$ of cyclic orders, i.e. a planar one.

- If $m>1$, we can multiply the numerator and denominator by $(a+1, \widehat{b})$ :

\section{Conclusion}

We have classified nonplanar on-shell diagrams according to whether they possess rational top forms, and we proved its equivalence to linear BCFW bridges. We conclude that when attaching linear bridges, geometric constraints of the nonpla-

$$
\begin{aligned}
\frac{1}{f(C)}= & \frac{(a, b-1)(a+1, \widehat{b})}{(12) \cdots(b-2, b-1)(b-1, \widehat{b})(a, \widehat{b})(b+1, b-1)(b+1, b+2) \cdots(n 1)(a+1, \widehat{b})} \\
= & \frac{(a, a+1)(\widehat{b}, b-1)+(a, \widehat{b})(a+1, b-1)}{(12) \cdots(b-2, b-1)(b-1, \widehat{b})(a, \widehat{b})(b+1, b-1)(b+1, b+2) \cdots(n 1)(a+1, \widehat{b})} \\
= & \frac{1}{(12) \cdots(a-1, a)(a+1, a+2) \cdots(b-2, b-1)(a, \widehat{b})(b-1, b+1)(b+1)(b+2) \cdots(n 1)(a+1, \widehat{b})} \\
& +\frac{(a+1, b-1)}{(12) \cdots(b-2, b-1)(b-1, \widehat{b})(a+1, \widehat{b})(b+1, b-1)(b+1, b+2) \cdots(n 1)} .
\end{aligned}
$$

The first term is already planar, while the second is not obvious.

- If $m=2$, the second term is planar.

- If $m>2$, we multiply the integrand by $(a+2, b),(a+$ $3, b), \ldots,(a+m-1, b)$ one by one. For each step of multiplication, we utilize the Pluck relation to transform the nonplanar term into a summation of planar terms and a remaining term. The final term left after series of multiplication is nar diagrams - tangled or untangled - can all be constructed systematically. With this chain of BCFW bridges rational top forms of the nonplanar on-shell diagrams can then be derived in a straightforward way. This method applies to leading singularities of nonplanar multi-loop amplitudes beyond MHV.

Acknowledgements GC thanks Nima Arkani-Hamed for helpful discussion and useful comments. We thank Peizhi Du, Shuyi Li and Hanqing Liu for constructive discussion. Yuan Xin thanks Bo Feng for introducing the background on the recent developments of scatter-

$\frac{(a+m-1, b-1)}{(12) \cdots(b-2, b-1)(b-1, \widehat{b})(a+m-1, \widehat{b})(b+1, b-1)(b+1, b+2) \cdots(n 1)}$.

Since $a+m=b-1$, this term is also planar.

Following these steps, we can finally simplify the top forms of all nonplanar MHV amplitudes into the sum of planar ones. One can easily verify that the simplification process from nonplanar one to planar term's summation is equivalent to applying the KK relation to the MHV amplitudes.

In this section, we construct the top forms of the nonplanar on-shell graphs. The key step is attaching a nonadjacent BCFW bridge to a planar diagram. The cyclic order of $f(C)$ is then broken and we obtain a different integrand from the planar ones. Keep attaching bridges on the identity and we can arrive at the top form of our target - the nonplanar leading singularity. We then break down the top forms of the nonplanar MHV amplitudes into a summation of the planar top forms. For the leading singularities of the one-loop amplitudes, this simplification is similar to the $\mathrm{KK}$ relation. For leading singularities of the general amplitudes, the relation between the top form's simplification and the KK relation will be discussed in our future work (Table 3). ing amplitude. GC, RX, and HZ have been supported by the Fundamental Research Funds for the Central Universities under contract 020414340080, NSF of China Grant under contract 11405084, the Open Project Program of State Key Laboratory of Theoretical Physics, Institute of Theoretical Physics, Chinese Academy of Sciences, China (No. Y5KF171CJ1). We also thank Y. Gao, T. Han for hospitality and the Key Laboratory of Theoretical Physics for hosting.

Open Access This article is distributed under the terms of the Creative Commons Attribution 4.0 International License (http://creativecomm ons.org/licenses/by/4.0/), which permits unrestricted use, distribution, and reproduction in any medium, provided you give appropriate credit to the original author(s) and the source, provide a link to the Creative Commons license, and indicate if changes were made. Funded by SCOAP ${ }^{3}$.

\section{Appendix}

All rational top forms can be constructed by our method described above. In our discussion we have assumed that the BCFW bridges are the linear BCFW bridges - each succes- 
sive $\alpha$-shift transforms the constraints linearly and can thus be represented by a rational function of minors of the underlying constraint $\mathrm{C}$-matrix. However, not all on-shell diagrams are made up completely of such bridges: the constraints can be nonlinear in alpha and cannot be written as rational functions under some shift. Such an on-shell diagram will not have a rational top form.

We present a counter example, $\mathcal{A}_{10}^{4}$. Upon attaching the bridge $(3,4)$, two constraints $(2,3,4,8)^{3}$ and $(1,3,4,6)^{3}$ emerge (the superscripts denote the number of independent column vectors), with two columns, 3 and 4 , being the same. Attaching the bridge (5,3), one of the constraints is removed and the other one becomes $((5,3) \cap(2,4,8), 1,4,6)^{3}$. If we go on attaching the bridge $(7,4)$, the tangled constraint is removed. However, due to the column 4 appearing twice in that constraint, such a bridge results in a nonlinear shift of the algebraic ideal. Therefore such a $\alpha$-shift cannot be represented linearly by some minor being zero, violating our linearity requirements in the construction of rational top forms.

\section{References}

1. R. Britto, F. Cachazo, B. Feng, Computing one-loop amplitudes from the holomorphic anomaly of unitarity cuts. Phys. Rev. D 71, 025012 (2005). arXiv:hep-th/0410179

2. R. Britto, F. Cachazo, B. Feng, New recursion relations for tree amplitudes of gluons. Nucl. Phys. B 715, 499-522 (2005). arXiv:hep-th/0412308

3. R. Britto, F. Cachazo, B. Feng, E. Witten, Direct proof of the treelevel scattering amplitude recursion relation in Yang-Mills theory. Phys. Rev. Lett. 94, 181602 (2005). arXiv:hep-th/0501052

4. B. Feng, M. Luo, An introduction to on-shell recursion relations. Front. Phys. 7, 533-575 (2012). arXiv: 1111.5759

5. Z. Bern, L. Dixon, D.L. Dunbar, D.A. Kosower, One-loop n-point gauge theory amplitudes, unitarity and collinear limits. Nucl. Phys. B 425, 217-260 (1994). arXiv:hep-ph/9403226

6. Z. Bern, L. Dixon, D.C. Dunbar, D.A. Kosower, Fusing gauge theory tree amplitudes into loop amplitudes. Nucl. Phys. B 435, 59101 (1995). arXiv:hep-ph/9409265

7. Z. Bern, L.J. Dixon, V.A. Smirnov, Iteration of planar amplitudes in maximally supersymmetric Yang-Mills theory at three loops and beyond. Phys. Rev. D 72, 085001 (2005). arXiv:hep-th/0505205

8. R. Britto, F. Cachazo, B. Feng, Generalized unitarity and one-loop amplitudes in $\mathrm{N}=4$ super-Yang Mills. Nucl. Phys. B 725, 275-305 (2005). arXiv:hep-th/0412103

9. R. Britto, E. Buchbinder, F. Cachazo, B. Feng, One-loop amplitudes of gluons in supersymmetric qcd. Phys. Rev. D 72, 065012 (2005)

10. E.I. Buchbinder, F. Cachazo, Two-loop amplitudes of gluons and octa-cuts in Script N = 4 super Yang-Mills. J. High Energy Phys. 11, 36 (2005). arXiv:hep-th/0506126

11. J. Drummond, J. Henn, G. Korchemsky, E. Sokatchev, Generalized unitarity for $\mathrm{N}=4$ super-amplitudes. Nucl. Phys. B 869, 452-492 (2013). arXiv:0808.0491

12. P. Mastrolia, E. Mirabella, G. Ossola, T. Peraro, Integrandreduction for two-loop scattering amplitudes through multivariate polynomial division. Phys. Rev. D 87, 085026 (2013). arXiv:1209.4319
13. P. Mastrolia, E. Mirabella, G. Ossola, T. Peraro, H. van Deurzen, The integrand reduction of one- and two-loop scattering amplitudes. PoS LL2012, 028 (2012). arXiv: 1209.5678

14. P. Mastrolia, E. Mirabella, G. Ossola, T. Peraro, Multiloop integrand reduction for dimensionally regulated amplitudes. arXiv: 1307.5832

15. H. van Deurzen, G. Luisoni, P. Mastrolia, E. Mirabella, G. Ossola, et al., Multi-loop integrand reduction via multivariate polynomial division. arXiv:1312.1627

16. Z. Bern, M. Czakon, D. Kosower, R. Roiban, V. Smirnov, Two-loop iteration of five-point $\mathrm{N}=4$ super-Yang-Mills amplitudes. Phys. Rev. Lett. 97, 181601 (2006). arXiv:hep-th/0604074

17. J.J. Carrasco, H. Johansson, Five-point amplitudes in $\mathrm{N}=4$ superYang-Mills theory and N = 8 supergravity. Phys. Rev. D 85, 025006 (2012). arXiv:1106.4711

18. Z. Bern, L. Dixon, D. Kosower, R. Roiban, M. Spradlin et al., The two-loop six-gluon MHV amplitude in maximally supersymmetric Yang-Mills theory. Phys. Rev. D 78, 045007 (2008). arXiv:0803.1465

19. B. Eden, G.P. Korchemsky, E. Sokatchev, More on the duality correlators/amplitudes. Phys. Lett. B 709, 247-253 (2012). arXiv: 1009.2488

20. B. Eden, P. Heslop, G.P. Korchemsky, E. Sokatchev, The supercorrelator/super-amplitude duality: Part I. Nucl. Phys. B 869, 329377 (2013). arXiv:1103.3714

21. B. Eden, P. Heslop, G.P. Korchemsky, E. Sokatchev, The supercorrelator/super-amplitude duality: Part II. Nucl. Phys. B 869, 378 416 (2013). arXiv: 1103.4353

22. F. Cachazo, Sharpening the leading singularity. ArXiv e-prints (2008). arXiv:0803.1988

23. N. Kanning, T. Lukowski, M. Staudacher, A shortcut to general tree-level scattering amplitudes in $\mathcal{N}=4 \mathrm{SYM}$ via integrability. Fortsch. Phys. 62, 556-572 (2014). arXiv: 1403.3382

24. A. Ochirov, Scattering amplitudes in gauge theories with and without supersymmetry. arXiv: 1409.8087

25. J. Broedel, M. de Leeuw, M. Rosso, A dictionary between Roperators, on-shell graphs and Yangian algebras. JHEP 1406, 170 (2014). arXiv:1403.3670

26. J. Broedel, M. de Leeuw, M. Rosso, Deformed one-loop amplitudes in $\mathrm{N}=4$ super-Yang-Mills theory. arXiv:1406.4024

27. N. Beisert, J. Broedel, M. Rosso, On Yangian-invariant regularization of deformed on-shell diagrams in $\mathcal{N}=4$ super-Yang-Mills theory. J. Phys. A: Math. Theor. 47(36), 365402 (2014)

28. D. Chicherin, S. Derkachov, R. Kirschner, Yang-Baxter operators and scattering amplitudes in super-Yang-Mills theory. Nucl. Phys. B 881, 467-501 (2014)

29. J. Drummond, J. Henn, G. Korchemsky, E. Sokatchev, Dual superconformal symmetry of scattering amplitudes in $\mathrm{N}=4$ super-YangMills theory. Nucl. Phys. B 828, 317-374 (2010). arXiv:0807.1095

30. J. Drummond, J. Henn, All tree-level amplitudes in N = 4 SYM. JHEP 0904, 018 (2009). arXiv:0808.2475

31. J.M. Drummond, J.M. Henn, J. Plefka, Yangian symmetry of scattering amplitudes in $\mathrm{N}=4$ super Yang-Mills theory. JHEP 0905, 046 (2009). arXiv:0902.2987

32. A. Brandhuber, P. Heslop, G. Travaglini, Proof of the dual conformal anomaly of one-loop amplitudes in N = 4 SYM. JHEP 0910, 063 (2009). arXiv:0906.3552

33. N. Arkani-Hamed, F. Cachazo, J. Kaplan, What is the simplest quantum field theory? J. High Energy Phys. 9, 16 (2010). arXiv:0808.1446

34. F. Cachazo, D. Skinner, On the structure of scattering amplitudes in $\mathrm{N}=4$ super Yang-Mills and $\mathrm{N}=8$ supergravity. ArXiv e-prints (2008). arXiv:0801.4574

35. Z. Bern, J.J.M. Carrasco, H. Johansson, D.A. Kosower, Maximally supersymmetric planar Yang-Mills amplitudes at five loops. Phys. Rev. D 76, 125020 (2007). arXiv:0705.1864 
36. M. Spradlin, A. Volovich, C. Wen, Three-loop leading singularities and BDS ansatz for five particles. Phys. Rev. D 78, 085025 (2008). arXiv:0808.1054

37. N. Arkani-Hamed, F. Cachazo, C. Cheung, J. Kaplan, The Smatrix in twistor space. J. High Energy Phys. 3, 110 (2010). arXiv:0903.2110

38. M.F. Paulos, B.U.W. Schwab, Cluster algebras and the positive Grassmannian. JHEP 1410, 31 (2014). arXiv: 1406.7273

39. Y. Bai, S. He, The amplituhedron from momentum twistor diagrams. arXiv:1408.2459

40. L. Ferro, T. Lukowski, M. Staudacher, $N=4$ scattering amplitudes and the deformed Grassmannian. arXiv:1407.6736

41. S. Franco, D. Galloni, A. Mariotti, J. Trnka, Anatomy of the amplituhedron. arXiv: 1408.3410

42. H. Elvang, Y.-T. Huang, C. Keeler, T. Lam, T.M. Olson et al., Grassmannians for scattering amplitudes in $4 \mathrm{~d} \mathcal{N}=4 \mathrm{SYM}$ and 3d ABJM. arXiv:1410.0621

43. N. Arkani-Hamed, J.L. Bourjaily, F. Cachazo, A.B. Goncharov, A. Postnikov et al., Scattering amplitudes and the positive Grassmannian. arXiv: 1212.5605

44. S. Franco, D. Galloni, A. Mariotti, The geometry of on-shell diagrams. arXiv: 1310.3820

45. N. Arkani-Hamed, J.L. Bourjaily, F. Cachazo, A. Post-nikov, J. Trnka (2014). arXiv:1412.8475 [hep-th]

46. S. Franco, D. Galloni, B. Penante, C. Wen, arXiv:1502.02034

47. H. Elvang, D.Z. Freedman, M. Kiermaier, Dual conformal symmetry of 1-loop NMHV amplitudes in N = 4 SYM theory. JHEP 1003, 075 (2010). arXiv:0905.4379

48. L. Dolan, C.R. Nappi, E. Witten, A relation between approaches to integrability in superconformal Yang-Mills theory. J. High Energy Phys. 10, 17 (2003). arXiv:hep-th/0308089

49. L. Dolan, C.R. Nappi, E. Witten, Yangian symmetry in D = 4 superconformal Yang-Mills theory, in Quantum Theory and Symmetries ed. by P.C. Argyres, T.J. Hodges, F. Mansouri, J.J. Scanio, P. Suranyi, L.C.R. Wijewardhana), pp. 300-315 (2004). arXiv:hep-th/0401243

50. N. Beisert, J. Broedel, M. Rosso, On Yangian-invariant regularisation of deformed on-shell diagrams in $\mathrm{N}=4$ super-Yang-Mills theory. ArXiv e-prints (2014). arXiv: 1401.7274

51. R. Frassek, N. Kanning, Y. Ko, M. Staudacher, Bethe ansatz for Yangian invariants: towards super Yang-Mills scattering amplitudes. Nucl. Phys. B 883, 373 (2014). arXiv:1312.1693

52. A. Amariti, D. Forcella, Scattering amplitudes and toric geometry. JHEP 1309, 133 (2013). arXiv:1305.5252

53. J.L. Bourjaily, S. Caron-Huot, J. Trnka, Dual-conformal regularization of infrared loop divergences and the chiral box expansion. ArXiv e-prints (2013). arXiv: 1303.4734

54. S. Caron-Huot, Superconformal symmetry and two-loop amplitudes in planar N = 4 super Yang-Mills. JHEP 1112, 066 (2011). arXiv: 1105.5606

55. J. Drummond, L. Ferro, The Yangian origin of the Grassmannian integral. JHEP 1012, 010 (2010). arXiv: 1002.4622

56. N. Beisert, J. Henn, T. McLoughlin, J. Plefka, One-loop superconformal and Yangian symmetries of scattering amplitudes in $\mathrm{N}=4$ super Yang-Mills. J. High Energy Phys. 4, 85 (2010). arXiv: 1002.1733

57. J. Drummond, L. Ferro, Yangians, Grassmannians and T-duality. JHEP 1007, 027 (2010). arXiv: 1001.3348

58. B. Feng, R. Huang, Y. Jia, Gauge amplitude identities by on-shell recursion relation in S-matrix program. Phys. Lett. B 695, 350-353 (2011). arXiv:1004.3417
59. L.F. Alday, J. Maldacena, A. Sever, P. Vieira, Y-system for scattering amplitudes. J. Phys. A 43, 485401 (2010). arXiv:1002.2459

60. L. Mason, D. Skinner, Dual superconformal invariance, momentum twistors and Grassmannians. JHEP 0911, 045 (2009). arXiv:0909.0250

61. N. Beisert, T-duality, dual conformal symmetry and integrability for strings on $\operatorname{AdS}(5) \times S^{* *} 5$. Fortsch. Phys. 57, 329-337 (2009). arXiv:0903.0609

62. A. Agarwal, N. Beisert, T. McLoughlin, Scattering in massdeformed Script N $=4$ Chern-Simons models. J. High Energy Phys. 6, 45 (2009). arXiv:0812.3367

63. T. Bargheer, N. Beisert, W. Galleas, F. Loebbert, T. McLoughlin, Exacting N = 4 superconformal symmetry. JHEP 0911, 056 (2009). arXiv:0905.3738

64. I. Adam, A. Dekel, Y. Oz, On integrable backgrounds self-dual under fermionic T-duality. J. High Energy Phys. 4, 120 (2009). arXiv:0902.3805

65. H. Elvang, Y.-T. Huang, Scattering amplitudes. arXiv:1308.1697

66. P. Benincasa, New structures in scattering amplitudes: a review. arXiv: 1312.5583

67. N. Beisert, C. Ahn, L.F. Alday, Z. Bajnok, J.M. Drummond, L. Freyhult, N. Gromov, R.A. Janik, V. Kazakov, T. Klose, G.P. Korchemsky, C. Kristjansen, M. Magro, T. McLoughlin, J.A. Minahan, R.I. Nepomechie, A. Rej, R. Roiban, S. Schäfer-Nameki, C. Sieg, M. Staudacher, A. Torrielli, A.A. Tseytlin, P. Vieira, D. Volin, K. Zoubos, Review of AdS/CFT integrability: an overview. Lett. Math. Phys. 99, 3-32 (2012). arXiv: 1012.3982

68. J. Drummond, Tree-level amplitudes and dual superconformal symmetry. J. Phys. A 44, 454010 (2011). arXiv:1107.4544

69. L.J. Dixon, Scattering amplitudes: the most perfect microscopic structures in the universe. J. Phys. A 44, 454001 (2011). arXiv: 1105.0771

70. N. Beisert, On Yangian symmetry in planar $\mathrm{N}=4 \mathrm{SYM}$. arXiv: 1004.5423

71. J. Bartels, L. Lipatov, A. Prygarin, Integrable spin chains and scattering amplitudes. J. Phys. A 44, 454013 (2011). arXiv:1104.0816

72. J.M. Henn, Dual conformal symmetry at loop level: massive regularization. J. Phys. A Math. Gen. 44, 4011 (2011). arXiv:1103.1016

73. T. Bargheer, N. Beisert, F. Loebbert, Exact superconformal and Yangian symmetry of scattering amplitudes. J. Phys. A Math. Gen. 44, 4012 (2011). arXiv: 1104.0700

74. R. Roiban, Review of AdS/CFT integrability, Chapter V. 1: Scattering amplitudes-a brief introduction. Lett. Math. Phys. 99, 455479 (2012). arXiv:1012.4001

75. J. Drummond, Review of AdS/CFT integrability, Chapter V. 2: Dual superconformal symmetry. Lett. Math. Phys. 99, 481-505 (2012). arXiv: 1012.4002

76. N.J. Mackay, Introduction to Yangian symmetry in integrable field theory. Int. J. Mod. Phys. A 20, 7189-7217 (2005). arXiv:hep-th/0409183

77. D. Bernard, An introduction to Yangian symmetries. Int. J. Mod. Phys. B 7, 3517-3530 (1993). arXiv:hep-th/9211133

78. J.M. Henn, J.C. Plefka, Scattering amplitudes in gauge theories. Lect. Notes Phys. 883, 1-195 (2014)

79. P. Du, G. Chen, Y.-K.E. Cheung, Permutation relations of generalized Yangian invariants, unitarity cuts, and scattering amplitudes. JHEP 1409, 115 (2014). arXiv:1401.6610

80. R. Kleiss, H. Kuijf, Multigluon cross sections and 5-jet production at hadron colliders. Nucl. Phys. B 312, 616-644 (1989) 